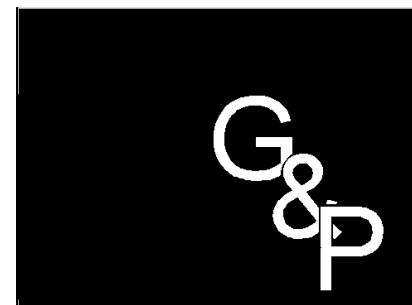

\title{
CUSTOS DA QUALIDADE E DA MANUFATURA: UM ESTUDO DE CASO NA INDÚSTRIA QUÍMICA
}

\section{GESTÃO $\&$ PRODUÇÃO}

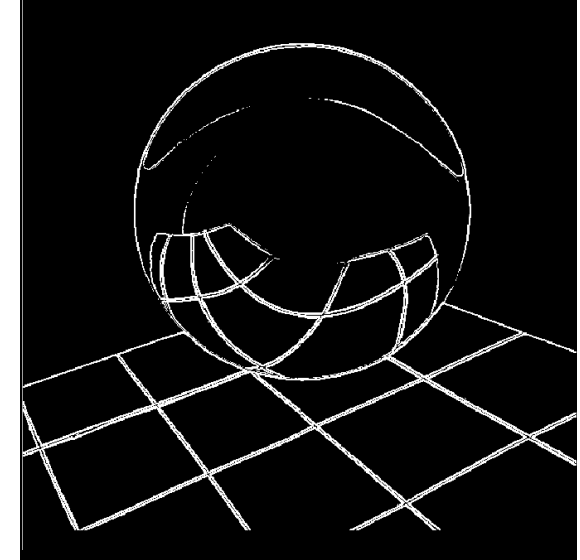

José Luis Duarte Ribeiro

Professor Doutor do Programa de Pós-Graduação em Engenharia de Produção Escola de Engenharia - UFRGS Praça Argentina $n^{\circ}$ 9, sala 402 - Porto Alegre - RS

Carla S. ten Caten Mestre e Doutoranda do Programa de Pós-Graduação em Engenharia Metalúrgica e Materiais Escola de Engenharia - UFRGS

v.3, n.3, p. 274-290, dez. 1996

\section{Resumo}

Este trabalho relata um estudo de caso envolvendo a otimização experimental de uma receita química. O estudo abrange as etapas de identificação do problema, planejamento do experimento, modelagem individual das variáveis de resposta, definição de uma função objetivo e otimização. Na identificação do problema e no planejamento do experimento foi utilizada uma estrutura matricial para reunir e organizar as informações. $O$ experimento contemplou cinco fatores controláveis e dez variáveis de resposta. Na etapa de modelagem individual, foram construídos modelos para a média e a variabilidade de cada uma das variáveis de resposta. A função objetivo utilizada no estudo de otimização foi a Função de Perda Quadrática Multivariada, proposta por Ribeiro \& Elsayed, acrescida dos custos de matéria prima e energia. Assim, a otimização foi conduzida levando em conta tanto os custos decorrentes da má qualidade (capturados pela função de perda) como os custos de matéria prima e energia. A otimização permitiu definir o melhor ajuste para os fatores controláveis. Ao final é feita uma análise de sensibilidade e é sugerido um envelope de operação para o controle do processo.

\section{Palavras-chave: qualidade, otimização, projeto de experimentos, regressão múltipla, função de perda.}




\section{Introdução}

$\mathrm{E}$ ste estudo de caso descreve um trabalho de otimização experimental da qualidade e do custo de uma receita química. $\mathrm{O}$ objetivo é quantificar o efeito dos fatores controláveis, neste caso, os componentes de uma receita, sobre as variáveis de resposta e, posteriormente, identificar o ajuste ótimo para os fatores controláveis. Como as informações são sigilosas, os dados foram alterados (mediante uma transformação linear) e no texto citam-se apenas fatores controláveis $X_{1}, \ldots, \quad X_{K} \quad$ e variáveis de resposta $Y_{1}, \ldots \ldots . Y_{J}$, sem indicar o nome das variáveis.

A otimização de produtos e processos é uma tarefa frequiente no meio industrial, pois pode implicar redução de custos ou melhoria da qualidade e da produtividade. A otimização busca identificar os ajustes dos fatores do processo que melhor atendem às variáveis de resposta de interesse para o clidunta vez ajustados os fatores do processo em seus níveis ótimos, os índices de refugo e retrabalho podem reduzir-se drasticamente, o que representa uma redução de custos. No entanto, a economia ainda maior está na redução dos custos da má qualidade, representados por custos de garantia, custos de reposição e até mesmo custo da eventual perda do cliente. Essa economia se materializa quando no processo de otimização se consegue um produto robusto, ou seja, um produto que atende aos valores nominais estabelecidos no projeto e que tem um bom desempenho no campo, mesmo quando submetido a condições adFensagedaluso. processo de otimização envolve múltiplas características de qualidade, pois o cliente valoriza diversos aspectos de um produto. Da mesma forma, para se atingir qualidade, é necessário satisfazer a múltiplos objetivos, tais como: minimizar os desvios do alvo, maximizar a robustez frente aos fatores de ruído e às oscilações dos fatores do processo. Esses três objetivos estão relacionados com a qualidade de um produto, pois sob a ótica atual, fabricar um produto de qualidade significa fabricar um produto centrado no valor nominal de projeto, com variabilidade reduzida em torno desse valor. Qualquer desvio do valor alvo representa uma perda de qualidade, e essa perda implica custos.

Outro objetivo muito importante, que pode ser incorporado no estudo de otimização, é a minimização dos custos da matéria-prima e da energia dispendidos na fabricação do produto. Dessa forma, o ajuste ótimo busca minimizar o conjunto dos custos gerados pela má qualidade do produto e pela sua fabricação.

Uma vez que o desempenho dos produtos e processos é medido por várias características de qualidade, alcançar os objetivos mencionados acima requer um compromisso que pondere as importâncias relativas das características de qualidade e considere os diferentes objetivos mencionados acima. Devido à complexidade do problema, é necessário um método quantitativo e genérico para solucioná-lo.

Neste processo de otimização, utilizou-se inicialmente uma estrutura matricial para a coleta e organização de informações a respeito do problema em estudo. Posteriormente, utilizou-se Projeto de Experimentos para estruturar a coleta de dados e a análise. Uma vez coletados os dados, utilizaram-se rotinas de regressão múltipla para fazer a modelagem individual das variáveis de resposta em função dos fatores controláveis. 
Conhecidos os modelos individuais das variáveis de resposta, definiu-se uma função objetivo a ser otimizada. Essa função objetivo é uma extensão da função de perda quadrática desenvolvida inicialmente por Taguchi. A função de perda representa a perda de qualidade ou perda financeira acarretada ao cliente, toda vez que as variáveis de resposta se distanciam dos seus valores alvo.

A função objetivo usada neste estudo incorporou dez variáveis de resposta de interesse para o cliente, buscando estabelecer um compromisso entre maximizar qualidade e reduzir custos de fabricação. Para maximizar a qualidade de um produto é necessário minimizar os desvios em relação ao valor nominal e maximizar a robustez frente aos fatores de ruído e às oscilações dos fatores conArqlánteieisda definição da função objetivo, utilizaram-se rotinas de programação linear para identificar o ajuste dos fatores controláveis que minimiza essa função objetivo. $\mathrm{O}$ ajuste que minimiza a função objetivo representa o ajuste ótimo, ou seja, os níveis dos fatores controláveis que melhor atendem ao conjunto de variáveis de resposta identificado. Resumindo, o ajuste ótimo representa o ajuste de menor perda financeira para o cliente.

Uma vez identificado o ajuste ótimo dos componentes da receita, desenvolveu-se um simulador, que incorpora todos os modelos previamente obtidos e estima o valor das variáveis de resposta para qualquer ajuste dos componentes da receita. Dessa forma, a empresa pode verificar previamente a qualidade de uma receita que tenha interesse em produzir. $\mathrm{O}$ simulador fornece também as estimativas dos valores das variáveis de resposta, cujos ensaios são feitos fora da empresa, a partir das variáveis de resposta cujos ensaios são realizados dentro da empresa. Isso foi possível, uma vez que foram identificadas algumas correlações significativas entre as variáveis de resposta.

\section{Identificação do Problema}

$\mathrm{N}$

o estudo de otimização da receita química aplicou-se inicialmente uma estrutura matricial, proposta por ECHEVESTE \& RIBEIRO (1995). A estrutura de matrizes permite reunir, organizar e documentar facilmente todas as informações existentes e necessárias sobre as variáveis em estudo, que geralmente se encontram dispersas entre os técnicos e engenheiros da empresa.

Com a aplicação da estrutura matricial foi possível inicialmente identificar quais as características de qualidade de interesse do cliente. Uma vez identificadas essas características, elegeram-se as variáveis de resposta que seriam usadas para quantificálas. Atribuiu-se um peso a cada uma das variáveis de resposta, ponderando a importância relativa daquela variável sobre a qualidade do produto como um todo. Além disso, identificaram-se os limites de especificação de cada variável de resposta. A Tabela 1 resume essas informações. 
Tabela 1: Descrição das Variáveis de Resposta

\begin{tabular}{|c|c|c|c|c|c|c|}
\hline $\begin{array}{c}\text { Variável de } \\
\text { Resposta }\end{array}$ & Imp. & Tipo de & Valor & Valor & \multicolumn{2}{|c|}{ Lim. de Esp. } \\
\cline { 6 - 7 } & Rel. & V. R. & Atual & Alvo & MIN & MAX \\
\hline Y1 & 2 & NOMINAL & 8.0 & 8.5 & 7.93 & 9.07 \\
\hline Y2 & 2 & NOMINAL & 85 & 85 & 74,2 & 95,8 \\
\hline Y3 & 3 & MENOR-M & 220 & 210 & - & 232,7 \\
\hline Y4 & 3 & NOMINAL & 27 & 30 & 27,02 & 32,98 \\
\hline Y5 & 4 & NOMINAL & 62 & 62 & 59,49 & 64,51 \\
\hline Y6 & 4 & NOMINAL & 1.137 & 1.13 & 1.125 & 1.135 \\
\hline Y7 & 5 & MENOR-M & 80 & 65 & - & 78 \\
\hline Y8 & 4 & MAIOR-M & 1300 & 1400 & 1231,69 & - \\
\hline Y9 & 3 & MAIOR-M & 1900 & 2400 & 2328,02 & - \\
\hline Y10 & 4 & MAIOR-M & 500 & 530 & 496,42 & - \\
\hline
\end{tabular}

Nota: O valor alvo teórico para características do tipo maior é melhor (ou menor é melhor) é infinito (ou zero). No entanto, na prática, existe em geral um limite superior (ou inferior) que, caso atingido ou ultrapassado, já satisfaz plenamente às expectativas do cliente. Na tentativa de melhor modelar a realidade, define-se esse limite superior (ou inferior) como o valor alvo, e considera-se que a partir do momento que esse valor for atingido ou ultrapassado, a perda experimentada pelo cliente será nula. Modelar a perda dessa forma, possibilita uma representação mais fiel da realidade, permitindo estabelecer um compromisso adequado entre as diversas características de qualidade. Observase que, se o valor alvo de uma característica de qualidade do tipo maior é melhor é considerado infinito, essa característica estará sempre longe do seu valor alvo, puxando o ajuste ótimo global nessa direção, em detrimento de outras características de qualidade importantes para o cliente.

\section{Planejamento e Execução do Experimento}

A inda trabalhando com a estrutura matricial, listaram-se todos os fatores do processo que tinham influência sobre as variáveis de resposta. Posteriormente, atribuíram-se pesos às relações entre eles, representando o grau de influência dos fatores do processo sobre cada uma das variáveis de resposta. Quanto maior a influência de um parâmetro sobre uma determinada variável de resposta, maior o peso daquela relação.

Logo após identificaram-se os intervalos de investigação dos fatores do processo e também a existência de possíveis interações entre eles. A existência de interações entre fatores do processo implica que o efeito de um fator do processo sobre a variável de resposta depende do nível em que se encontra um outro fator do processo.

Uma vez documentadas todas essas informações para cada um dos fatores do processo, escolheram-se os fatores controláveis, ou seja, aquele subconjunto dos fatores do processo que seriam ensaiados no experimento. A escolha foi feita dando-se prioridade aos fatores do processo que exercem maior efeito sobre as variáveis de resposta mais importantes. 
Vale ressaltar que todas essas informações são necessárias para o correto planejamento do experimento e, posteriormente, para o processo de otimização global do produto. Mais detalhes sobre as fases de coleta de informações e

planejamento do experimento podem ser vistos em COLEMANN \& MONTGOMERY (1993) e em HAHN (1977). A Tabela 2 resume as informações coletadas sobre os fatores controláveis.

Tabela 2: Descrição dos Fatores Controláveis

\begin{tabular}{|c|c|c|c|c|}
\hline \multirow{2}{*}{$\begin{array}{c}\text { Fatores } \\
\text { Controláveis }\end{array}$} & \multirow{2}{*}{$\begin{array}{c}\text { Ajuste } \\
\text { Atual } \\
\end{array}$} & \multicolumn{2}{|c|}{ Intervalo de Ajuste } & \multirow{2}{*}{$\begin{array}{r}\text { Facilidade } \\
\text { de Ajuste } \\
\end{array}$} \\
\hline & & Mínimo & Máximo & \\
\hline $\mathrm{X} 1$ & 4,0 & 3,0 & 5,0 & 10 \\
\hline $\mathrm{X} 2$ & 6,5 & 5,5 & 6,5 & 10 \\
\hline X3 & 19,5 & 18 & 22 & 10 \\
\hline $\mathrm{X} 4$ & 3,5 & 2,0 & 4,0 & 10 \\
\hline $\mathrm{X} 5$ & 8 & 8 & 12 & 10 \\
\hline
\end{tabular}

A facilidade de ajuste dos fatores do processo é questionada junto aos engenheiros com o objetivo de identificar quais os fatores mais difíceis de ajustar, ou seja, quais os fatores cujo setup é muito difícil de ser modificado. Utilizou-se uma escala de 5-10 onde o valor 5 representa um fator muito difícil de ajustar e o valor 10 representa um fator fácil de ajustar. A informação referente à facilidade de ajuste é importante na atribuição de prioridade aos fatores do processo a serem incluídos no experimento e na garantia do caráter aleatório do experimento. Neste experimento todos os fatores eram fáceis de ajustar (valor 10), o que permitiu que ele fosse completamente aleatório.
Uma vez reunido todo o conhecimento prévio dos engenheiros a respeito do processo, e levando-se em consideração algumas restrições experimentais, planejouse o estudo experimental. O planejamento foi realizado utilizando-se o método de Projeto de Experimentos, introduzido por Fischer em 1935, que se apóia em conceitos estatísticos. Esse método permite estruturar uma seqüência de ensaios que atenda aos objetivos pré-estabelecidos pelos engenheiros de maneira confiável, eficiente e econômica. Mais detalhes sobre Projeto de Experimentos podem ser vistos em BOX \& DRAPPER (1987), BOX, HUNTER \& HUNTER (1978), MONTGOMERY (1991) ou em NANNI \& RIBEIRO (1991).

Tabela 3: Matriz Experimental

\begin{tabular}{|c|c|c|c|c|c|}
\hline Num & X1 & X2 & X3 & X4 & X5 \\
\hline 1 & 3,0 & 5,5 & 18 & 4,0 & 8 \\
\hline 2 & 3,0 & 6,5 & 18 & 2,0 & 8 \\
\hline 3 & 3,0 & 5,5 & 18 & 2,0 & 12 \\
\hline 4 & 3,0 & 6,5 & 18 & 4,0 & 12 \\
\hline 5 & 3,0 & 5,5 & 22 & 2,0 & 8 \\
\hline 6 & 3,0 & 6,5 & 22 & 4,0 & 8 \\
\hline
\end{tabular}




\begin{tabular}{|c|c|c|c|c|c|}
\hline 7 & 3,0 & 5,5 & 22 & 4,0 & 12 \\
\hline 8 & 3,0 & 6,5 & 22 & 2,0 & 12 \\
\hline 9 & 5,0 & 5,5 & 18 & 2,0 & 8 \\
\hline
\end{tabular}

Tabela 3: Matriz Experimental (Continuação)

\begin{tabular}{|c|c|c|c|c|c|}
\hline Num & $\mathbf{X 1}$ & $\mathbf{X 2}$ & $\mathbf{X 3}$ & $\mathbf{X 4}$ & $\mathbf{X 5}$ \\
\hline 10 & 5,0 & 6,5 & 18 & 4,0 & 8 \\
\hline 11 & 5,0 & 5,5 & 18 & 4,0 & 12 \\
\hline 12 & 5,0 & 6,5 & 18 & 2,0 & 12 \\
\hline 13 & 5,0 & 5,5 & 22 & 4,0 & 8 \\
\hline 14 & 5,0 & 6,5 & 22 & 2,0 & 8 \\
\hline 15 & 5,0 & 5,5 & 22 & 2,0 & 12 \\
\hline 16 & 5,0 & 6,5 & 22 & 4,0 & 12 \\
\hline 17 & 4,0 & 6,0 & 20 & 3,0 & 10 \\
\hline 18 & 4,0 & 6,0 & 20 & 3,0 & 10 \\
\hline 19 & 4,0 & 6,0 & 20 & 3,0 & 10 \\
\hline 20 & 4,0 & 6,0 & 20 & 3,0 & 10 \\
\hline 21 & 4,0 & 6,0 & 20 & 3,0 & 10 \\
\hline 22 & 4,0 & 6,0 & 20 & 3,0 & 10 \\
\hline 23 & 4,0 & 6,0 & 20 & 3,0 & 10 \\
\hline 24 & 4,0 & 5,5 & 20 & 3,0 & 10 \\
\hline 25 & 3,0 & 6,0 & 20 & 3,0 & 10 \\
\hline 26 & 5,0 & 6,0 & 20 & 3,0 & 10 \\
\hline 27 & 4,0 & 6,5 & 20 & 3,0 & 10 \\
\hline
\end{tabular}

Os dezesseis primeiros ensaios da matriz experimental correspondem a um projeto fatorial fracionado $2^{5-1}$. Para permitir um teste referente ao ajuste do modelo linear, foram acrescentados sete pontos centrais. Os últimos quatro ensaios foram realizados em níveis intermediários em duas direções $\mathrm{X} 1 \mathrm{e}$ $\mathrm{X} 2$ (face do cubo) mantendo os demais fatores nos níveis centrais, pois os engenheiros acreditavam na hipótese de efeitos quadráticos dos fatores controláveis $\mathrm{X} 1$ e X2 sobre as variáveis de resposta. A ordem de realização dos tratamentos foi tornada aleatória, de forma a distribuir o efeito dos fatores não-controláveis. A Tabela 3 mostra a matriz experimental com os tratamentos realizados (em ordem não ale@ómanhecimento prévio dos engenheiros a respeito do processo em estudo indicava que os fatores controláveis afetava a variabilidade. Assim, fez-se a modelagem da variabilidade seguindo um método iterativo explicado a seguir.

Primeiramente, utilizando-se regressão múltipla, fez-se uma modelagem individual das médias de cada variável de resposta, incluindo termos lineares, termos de interação e termos quadráticos em X1 e X2.

Como o experimento realizado não contemplava repetições, a modelagem da variabilidade das variáveis de resposta foi realizada baseada na amplitude dos resíduos (os resíduos se referem a diferenças entre os valores observados e aqueles estimados pelo modelo das médias). Para cada variável de resposta traçaram-se os gráficos de resíduos relativos a cada um dos fatores controláveis, e mediu-se a variância dos resíduos nos níveis extremos do fator em questão. 
Uma vez estimada a variância para cada nível do fator controlável, usou-se o teste $\mathrm{F}$ para verificar se havia diferenças significativas entre as variâncias observadas nos níveis extremos. Caso as variâncias apresentassem diferença significativa, acrescentava-se ao modelo de variabilidade um termo linear correspondendo ao fator em questão. Se as variâncias não apresentavam diferença significativa, o termo não era acrescentado ao modelo.

Conhecidos os modelos de variabilidade, aplicou-se uma Regressão Múltipla Ponderada, em que os pesos utilizados foram proporcionais ao inverso da variância. Os modelos obtidos por meio da Regressão Múltipla Ponderada são os modelos apresentados neste artigo. Nota-se que após ajustados os modelos, por meio da Regressão Múltipla Ponderada, fez-se novamente a análise de resíduos para revisar os modelos de variabilidade. Os modelos de variabilidade apresentados neste artigo são os modelos revisados.

$\mathrm{Na}$ modelagem das variáveis de resposta, trabalhou-se com níveis codificados dos fatores controláveis, pois isso facilita a análise estatística dos dados. Apresenta-se a seguir a fórmula para converter os níveis reais (NR) em níveis codificados (NC):

$$
N C=\frac{N R-M I}{((L S I-L I I) / 2)}
$$

onde: $M I$ representa a média do intervalo investigado;

LSI representam o limite superior do intervalo investigado.

LII representam o limite inferior do intervalo investigado.

Tabela 4: Níveis Reais e Codificados dos Fatores Controláveis

\begin{tabular}{|c|c|c|}
\hline Fatores controláveis & Níveis reais & Níveis codificados \\
\hline $\mathrm{X} 1$ & $3,0 \Leftrightarrow 5,0$ & $-1 \Leftrightarrow 1$ \\
\hline $\mathrm{X} 2$ & $5,5 \Leftrightarrow 6,5$ & $-1 \Leftrightarrow 1$ \\
\hline $\mathrm{X} 3$ & $18,0 \Leftrightarrow 22,0$ & $-1 \Leftrightarrow 1$ \\
\hline $\mathrm{X} 4$ & $2,0 \Leftrightarrow 4,0$ & $-1 \Leftrightarrow 1$ \\
\hline $\mathrm{X} 5$ & $8,0 \Leftrightarrow 12,0$ & $-1 \Leftrightarrow 1$ \\
\hline
\end{tabular}

\section{Modelagem Individual das Variáveis de Resposta}

$\mathrm{N}$

esta etapa, usando o procedimento iterativo descrito na seção 2, baseado no uso de Regressão Múltipla, modelou-se cada variável de resposta em função dos fatores controláveis. Os modelos permitem quantificar o efeito dos fatores controláveis. Dessa forma, sabe-se quais os fatores que exercem maior efeito sobre cada uma das variáveis de resposta. A seguir são listados todos os modelos obtidos para a média e a variabilidade de cada variável de resposta. Foram mantidos nos modelos os coeficientes significativos a um nível de significância $\alpha=5 \%$.

Modelos para a média e a variabilidade de Y1 (níveis codificados)

$Y 1=7,10+1,08 * X 1+0,64 * X 1^{2}+1,11 * X 2+0,54 * X 2^{2}+0,42 * X 4+0,256 * X 1 * X 2 ; \quad \boldsymbol{R}^{2}=\mathbf{0 , 9 1}$ 
$\sigma_{Y 1}=0,362+0,186 * X 1+0,118 * X 2$

Modelos para a média e a variabilidade de Y2 (níveis codificados):

$Y 2=74,62-2,33 * X 1-6,26 * X 2^{2} ; \quad \boldsymbol{R}^{2}=\mathbf{0 , 3 9}$

$\sigma_{Y 2}=4,125-1,40 * X 3+1,58 * X 5$

Y 1

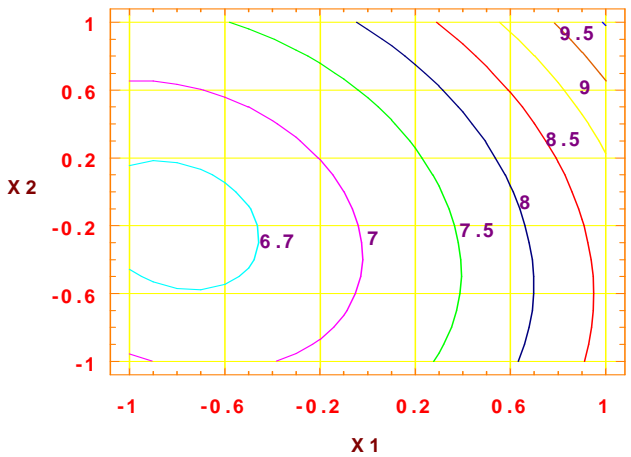

Fig. 1: Isovalores para Y1
Y 2

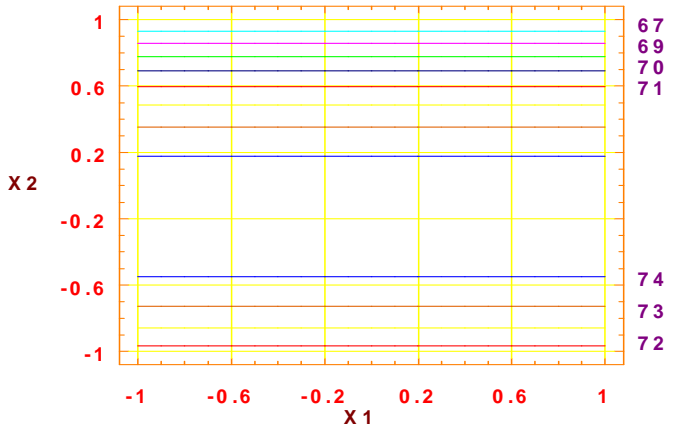

Fig. 2: Isovalores para Y2

Modelos para a média e a variabilidade de Y3 (níveis codificados)

$Y 3=201,19-4,89 * X 1-7,78 * X 1^{2}-3,89 * X 2-9,88 * X 5 ; \quad \boldsymbol{R}^{2}=\mathbf{0 , 7 9}$

$\sigma_{Y 3}=6,225+2,525 * X 1$

Modelos para a média e a variabilidade de Y4 (níveis codificados)

$Y 4=31,57+3,60 * X 1+1,43 * X 1^{2}+1,98 * X 2+1,58 * X 2^{2}+1,69 * X 3+1,10 * X 4+2,36 * X 5 ; \boldsymbol{R}^{2}=\mathbf{0 , 9 3}$ $\sigma_{Y 4}=0,623+0,253 * X 2$

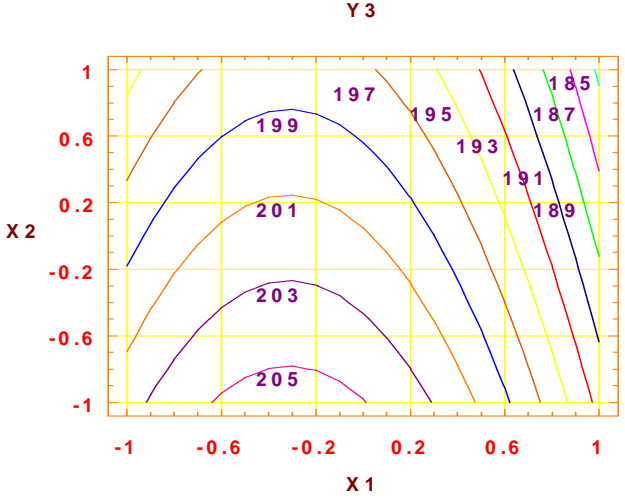

Fig. 3: Isovalores de Y3

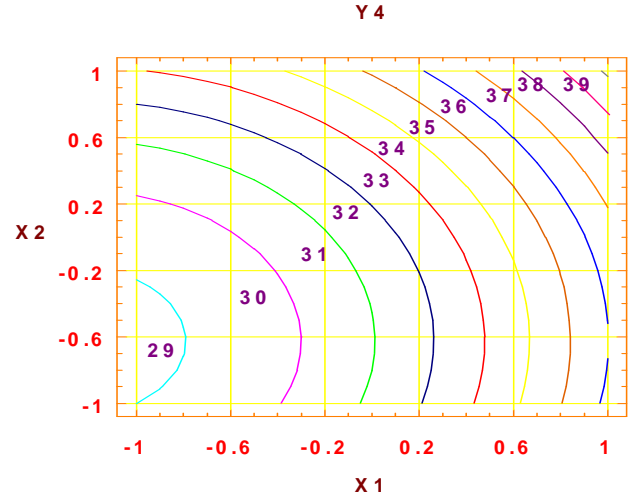

Fig. 4: Isovalores de Y4

Modelos para a média e a variabilidade de Y5 (níveis codificados)

$Y 5=61,73+2,06 * X 1+2,46 * X 1^{2}+2,33 * X 2+0,938 * X 3+0,938 * X 5 ; \quad \boldsymbol{R}^{2}=\mathbf{0 , 8 2}$

$\sigma_{Y 5}=1,633+0,892 * X 1$

Modelos para a média e a variabilidade de Y6 (níveis codificados)

$Y 6=1,132+0,00678 * X 1+0,0058 * X 1^{2}+0,0108 * X 2+0,0063 * X 2^{2}-0,00281 * X 4 ; \boldsymbol{R}^{2}=\mathbf{0 , 8 6}$

$\sigma_{Y 6}=0,00356+0,00202 * X 1+0,00825 * X 4$ 


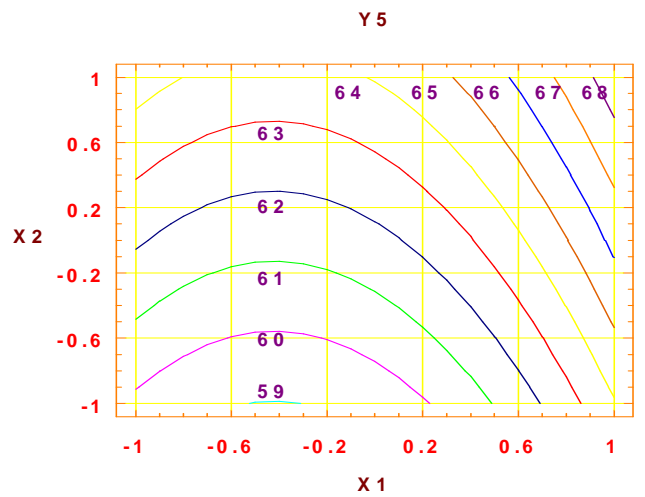

Fig. 5: Isovalores para Y5

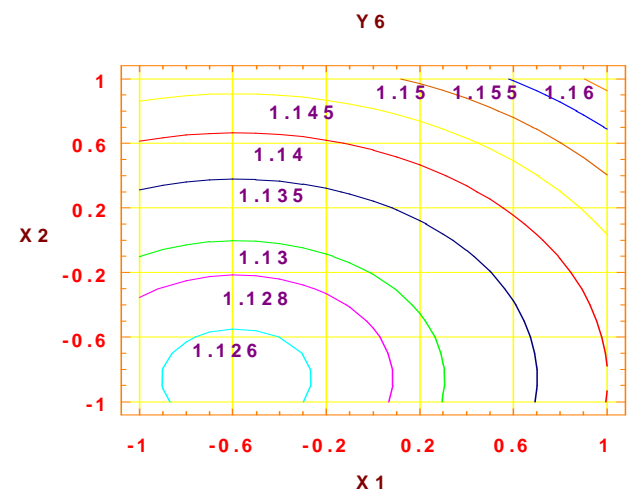

Fig. 6: Isovalores para Y6

Modelos para a média e a variabilidade de Y7 (níveis codificados)

$Y 7=74,11-1,17 * X 1-4,88 * X 4+1,47 * X 5+0,92 * X 1 * X 2-0,689 * X 3 * X 4 ; \boldsymbol{R}^{2}=\mathbf{0 , 9 2}$

$\sigma_{Y 7}=0,5$

Modelos para a média e a variabilidade de Y8 (níveis codificados)

$Y 8=1602+335,56 * X 1+179,47 * X 1^{2}+228,67 * X 2+154,47 * X 2^{2}+167,75 * X 3+125,75 * X 5 ; \boldsymbol{R}^{2}=\mathbf{0 , 9 1}$ $\sigma_{Y 8}=74,92+26,095 * X 2$

Y 7

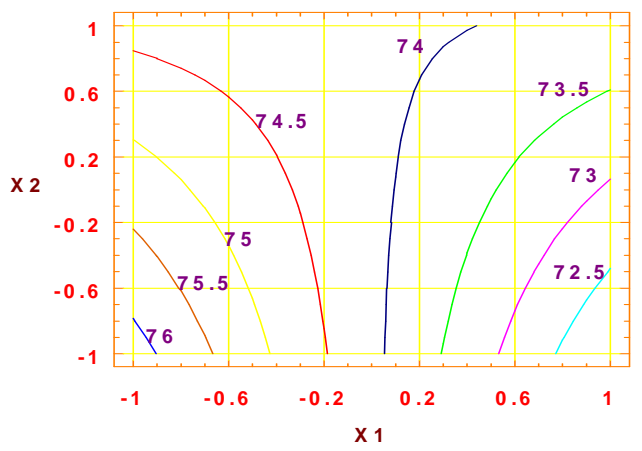

Fig. 7: Isovalores para Y7

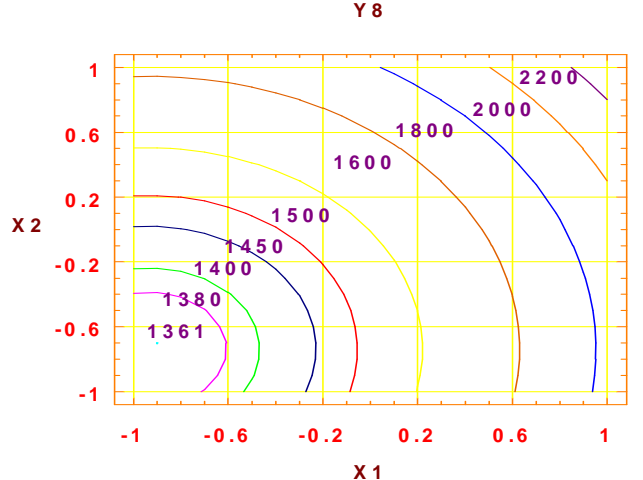

Fig. 8: Isovalores para Y8

Modelos para a média e a variabilidade de Y9 (níveis codificados)

$Y 9=3306,4-76,24 * X 1^{2}-123,44 * X 4+41,19 * X 1 * X 2 ; \boldsymbol{R}^{2}=\mathbf{0 , 7 5}$

$\sigma_{Y 9}=53,03-23,56 * X 1+18,52 * X 2$

Modelos para a média e a variabilidade de Y10 (níveis codificados)

$Y 10=520,7-58,06 * X 1-32,57 * X 1^{2}-34,17 * X 2-22,57 * X 2^{2}-32,69 * X 3-12,06 * X 4-21,56 * X 5 ; \boldsymbol{R}^{2}=\mathbf{0 , 9 3}$ $\sigma_{Y 10}=13,329-6,566 * X 2-6,673 * X 3$ 


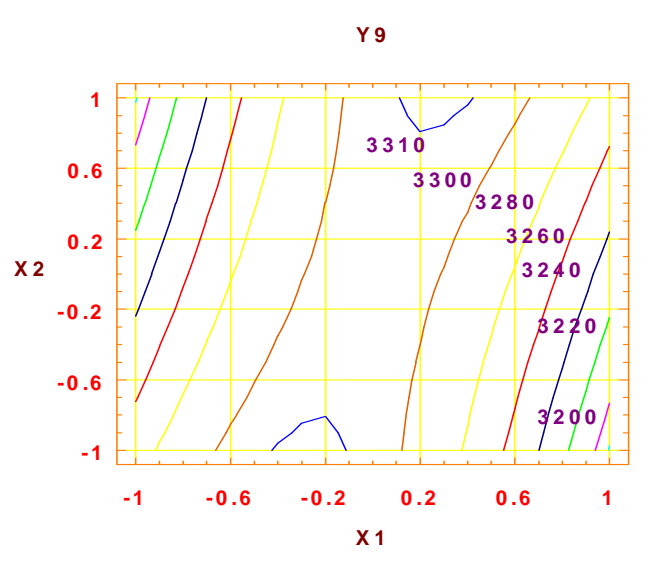

Fig. 9: Isovalores para Y9

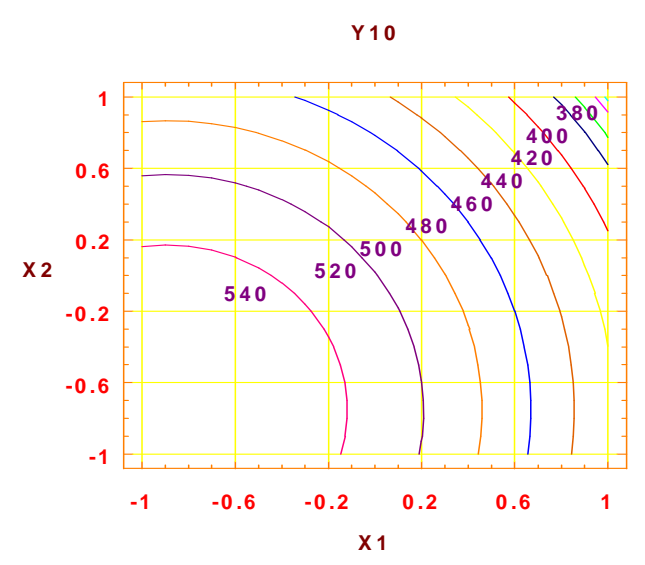

Fig. 10: Isovalores para Y10
A modelagem individual das variáveis de resposta efetuada anteriormente, refere-se a receitas cujas misturas foram realizadas em laboratório. Assim, os modelos obtidos estimam a resposta associada a uma receita produzida em laboratório.

Posteriormente, realizaram-se quatro misturas no laboratório e outras quatro no chão-de-fábrica, com o mesmo ajuste dos componentes da receita e com os mesmos lotes de matéria-prima.

Comparando-se as variáveis de resposta obtidas em laboratório com as obtidas no chão-de-fábrica, verificou-se um efeito de scale-up, ou seja, os resultados obtidos em laboratório não são exatamente iguais àqueles obtidos, com a mesma receita, no chão-de-fábrica.

Uma vez verificado o efeito de scale-up, estimou-se para cada variável de resposta a relação existente entre os resultados de laboratório e de chão-de-fábrica. A partir dessa relação, conhecidos os resultados de uma receita produzida no laboratório, pode-se prever o provável desempenho dessa receita quando produzida no chão-de-fábrica.

Os modelos que relacionam os resultados de chão-de-fábrica $\left(\mathrm{Yj}_{\mathrm{p}}\right)$ com aqueles obtidos em laboratório (Yj) são os seguintes:

\begin{tabular}{ll}
\hline $\mathrm{Y} 1_{\mathrm{P}}=1,06 * \mathrm{Y} 1$ & $\mathrm{Y} 6_{\mathrm{P}}=0,997 * \mathrm{Y} 6$ \\
$\mathrm{Y} 2_{\mathrm{P}}=1,16 * \mathrm{Y} 2$ & $\mathrm{Y} 7_{\mathrm{P}}=1,066 * \mathrm{Y} 7$ \\
$\mathrm{Y} 3_{\mathrm{P}}=1,06 * \mathrm{Y} 3$ & $\mathrm{Y} 8_{\mathrm{P}}=0,832 * \mathrm{Y} 8$ \\
$\mathrm{Y} 4_{\mathrm{P}}=0,96 * \mathrm{Y} 4$ & $\mathrm{Y} 9_{\mathrm{P}}=0,93 * \mathrm{Y} 9$ \\
$\mathrm{Y} 5_{\mathrm{P}}=1,01 * \mathrm{Y} 5$ & $\mathrm{Y} 10_{\mathrm{P}}=1,08 * \mathrm{Y} 10$ \\
\hline
\end{tabular}

O uso desses modelos é feito da seguinte maneira: primeiramente, estimam-se as variáveis de resposta (por exemplo Y1) para receitas produzidas em laboratório. Em seguida, usando as equações listadas acima, corrigem-se as estimativas das variáveis de resposta obtendo-se o provável desempenho da receita caso produzida no chão-de-fábrica (por exemplo $\mathrm{Y} 1_{\mathrm{P}}=1,06 \mathrm{Y} 1$ ).

Cabe ressaltar que há muitas vantagens em otimizar a receita em laboratório. Os experimentos de laboratório são mais fáceis de realizar, são mais fáceis de controlar, o erro experimental é menor, o custo e o tempo de execução são consideravelmente menores. 


\section{Definição da Função Objetivo}

A

função objetivo utilizada na otimização foi a Função de Perda Quadrática Multivariada (ver RIBEIRO \& ELSAYED, 1995 ou
CATEN \& RIBEIRO, 1995) acrescida de um termo referente aos custos de matéria prima e energia, uma vez que havia interesse em considerar esses aspectos.

\section{Função de Perda Quadrática Multivariada}

$$
\hat{Z}(i)=\sum_{j=1}^{J} w_{j}\left[\left(\hat{Y}_{j}-T_{j}\right)^{2}+\hat{\sigma}_{Y j}^{2}+\sum_{k=1}^{K} \hat{\sigma}_{X k}^{2}\left(\frac{\partial \hat{Y}_{j}}{\partial X_{k}}\right)^{2}\right]
$$

onde:

$\hat{Z}(i) \quad$ é o valor da função objetivo a ser minimizada; i refere-se a um certo tratamento, isto é, um certo ajuste dos fatores;

$w_{j} \quad$ são pesos que levam em conta as unidades e a importância relativa de cada variável de resposta;

$T_{j}^{T_{j}}, \sigma_{\mathrm{Yj}}$ é o valor alvo para a variável de resposta j;

$\sigma_{\mathrm{Xk}}$ estimativas da média e do desvio-padrão da variável de resposta j;

Para esse estudo, os pesos $w_{j}$ foram definidos levando-se em consideração a importância relativa $(I R)$ de cada variável de resposta e a semi-amplitude do intervalo de especificação $(\Delta E)$.

$$
w_{j}=\frac{I R_{j}}{\Delta E_{j}^{2}}
$$

Com respeito às oscilações dos fatores controláveis, os engenheiros indicaram que estas poderiam ser representadas por

\begin{tabular}{ccc}
\hline Coeficiente de Variação & $\begin{array}{c}\text { Desvio-padrão } \\
\text { (níveis reais) }\end{array}$ & $\begin{array}{c}\text { Desvio-padrão } \\
\text { (níveis codificados) }\end{array}$ \\
\hline$C V_{X 1}=4,0 \%$ & $\hat{\sigma}_{X 1}=0,04 \times 4=0,16$ & $\hat{\sigma}_{X 1}=\frac{0,16}{1}=0,16$ \\
$C V_{X 2}=0,5 \%$ & $\hat{\sigma}_{X 2}=0,005 \times 6=0,03$ & $\hat{\sigma}_{X 2}=\frac{0,03}{0,5}=0,06$ \\
$C V_{X 3}=0,5 \%$ & $\hat{\sigma}_{X 3}=0,005 \times 20=0,10$ & $\hat{\sigma}_{X 3}=\frac{0,10}{2}=0,05$ \\
$C V_{X 4}=4,0 \%$ & $\hat{\sigma}_{X 4}=0,04 \times 3=0,12$ & $\hat{\sigma}_{X 4}=\frac{0,12}{1}=0,12$ \\
$C V_{X 5}=4,0 \%$ & $\hat{\sigma}_{X 5}=0,04 \times 10=0,4$ & $\hat{\sigma}_{X 5}=\frac{0,4}{2}=0,2$ \\
\hline
\end{tabular}

Nota: o desvio padrão em níveis codificados é obtido dividindo-se o desvio-padrão em níveis reais pela semi-amplitude da faixa de variação do fator correspondente. 
Em sua forma original, a Função de Perda Quadrática Multivariada fornece valores que são proporcionais à perda financeira devida a má qualidade. Para obter a perda financeira em valores monetários, é preciso conhecer a constante de proporcionalidade $K$. O valor de $K$ para esse produto foi definido da seguinte maneira: a empresa oferece aos seus clientes duas categorias de produto, classificadas de acordo com a qualidade e com preços diferenciados de venda. Calculou-se o valor da perda $Z$ para um produto da categoria A (alta qualidade), sendo esse valor 64 unidades de perda. Calculou-se o valor da perda $Z$ (considerando os mesmos valores alvo da categoria A) para um produto da categoria $\mathrm{B}$ (baixa qualidade), sendo esse valor 179 unidades de perda.

O preço de venda do produto da categoria A $(\mathrm{R} \$ 1,42 / \mathrm{Kg})$ é $33 \%$ superior ao preço de venda do produto da categoria $B$ (R\$ 1,07/Kg). Dessa forma, há informações suficientes para calcular o valor da constante de proporcionalidade $K$.

$$
K=\frac{\Delta R \$}{\Delta Z}=\frac{(1,42-1,07)}{(179-64)}=0,003043
$$

Uma vez obtido o valor de $K$, pode-se transformar o valor da perda $\mathrm{Z}$ em unidades monetárias, ou seja, em custos da má qualidade:

$$
C_{Q}(i)=K \times \hat{Z}(i)=0,003043 \times \hat{Z}(i)
$$

Por outro lado, modelaram-se os custos de matéria-prima e energia, para a fabricação de um produto, em função dos fatores controláveis:

$$
\begin{array}{r}
C_{M}(i)=1,42+0,0117 * \mathrm{X} 1-0,0156 * \mathrm{X} 2+ \\
+0,00875 * \mathrm{X} 4+0,00375 * \mathrm{X} 5
\end{array}
$$

Com essas informações é possível trabalhar com o custo global:

$$
C_{G}(i)=C_{Q}(i)+C_{M}(i)
$$

Dessa forma, o ajuste ótimo encontrado é um compromisso entre custos devidos a má qualidade de um produto e custos da matéria-prima e da energia gastos na fabricação do produto.

\section{Otimização}

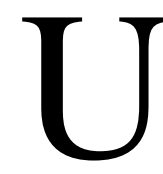

ma vez definida a função objetivo, utilizaram-se rotinas de programação linear para determinar o ajuste dos fatores controláveis que minimiza a função objetivo. $\mathrm{O}$ ajuste que minimiza a função objetivo é o que melhor atende simultaneamente ao conjunto das variáveis de resposta da produção, considerando-se qualidade e custos de manufatura.

A Tabela 5 apresenta o ajuste ótimo para os fatores controláveis, ou seja, para os componentes da receita.

Tabela 5: Ajuste Ótimo dos Fatores Controláveis

\begin{tabular}{|c|c|c|}
\hline Fatores controláveis & Níveis codificados & Níveis reais \\
\hline X1 & $-0,645$ & 3,36 \\
\hline X2 & 0,475 & 6,24 \\
\hline X3 & 0,955 & 21,9 \\
\hline X4 & 1,00 & 4,00
\end{tabular}




\begin{tabular}{|c|c|c|}
\hline $\mathrm{X} 5$ & $-1,00$ & 8,00 \\
\hline
\end{tabular}

Este ajuste dos fatores controláveis incorre em um custo global de R\$ 1,598 das variáveis de resposta que aparecem na unidades monetárias e fornece as estimativas Tabela 6.

Tabela 6: Variáveis de Resposta Referentes ao Ajuste Ótimo dos Fatores Controláveis

\begin{tabular}{|c|c|c|c|c|c|}
\hline $\begin{array}{c}\text { Variáveis de } \\
\text { resposta }\end{array}$ & Especificações & Valores alvo & $\begin{array}{c}\text { Valores } \\
\text { laboratório }\end{array}$ & $\begin{array}{c}\text { Valores } \\
\text { produção }\end{array}$ & Unidades \\
\hline Y1 & $7,5-9,5$ & 8,5 & 7,66 & 8,12 & u.m. \\
\hline Y2 & $75-95$ & 85 & 72,10 & 83,64 & u.m. \\
\hline Y3 & -230 & 210 & 209,14 & 221,7 & u.m. \\
\hline Y4 & $27-33$ & 30 & 31,49 & 30,23 & u.m. \\
\hline Y5 & $60-64$ & 62 & 62,49 & 63,11 & u.m. \\
\hline Y6 & $1,125-1,130$ & 1,13 & 1,134 & 1,130 & u.m. \\
\hline Y7 & -85 & 65 & 67,57 & 71,97 & u.m. \\
\hline Y8 & $900-$ & 1400 & 1638 & 1363 & u.m. \\
\hline Y9 & $2300-$ & 2400 & 3139 & 2919 & u.m. \\
\hline Y10 & $500-$ & 530 & 501,5 & 541,7 & u.m. \\
\hline
\end{tabular}

Em função do número excessivo de termos da função objetivo, ajustou-se um meta-modelo (um modelo do modelo) aos resultados gerados pela função objetivo. $\mathrm{O}$ meta-modelo, relativamente mais simples, facilita a análise gráfica dos resultados.

Uma vez obtido o meta-modelo, seu gráfico foi traçado em função de X1 e X2, que são os dois fatores controláveis com maior influência sobre a função objetivo. A

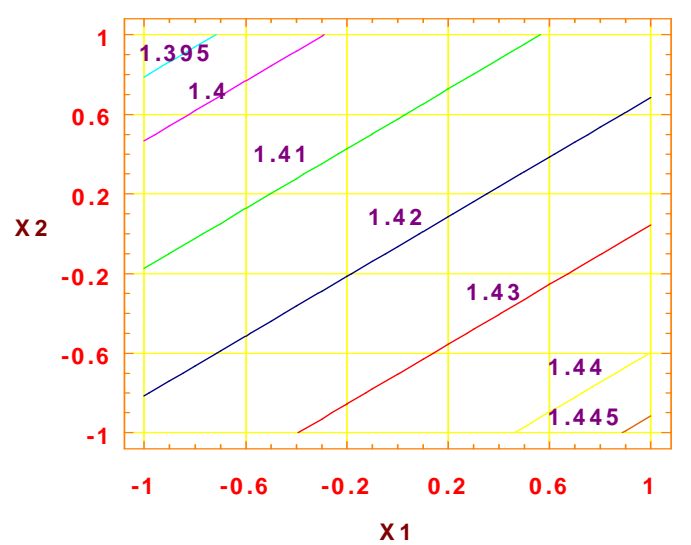

Fig. 11: Curvas de isovalores do custo de matéria-prima
Fig. 13 apresenta curvas de isovalores da função objetivo (custos globais) em função dos fatores X1 e X2, mantendo-se os fatores X3, X4 e X5 em seus ajustes ótimos.

Nessa figura pode-se identificar um envelope operacional para os fatores controláveis. Esse envelope define os limites de uma região dentro da qual os fatores controláveis $\mathrm{X} 1$ e $\mathrm{X} 2$ podem variar sem incorrer em maiores perdas para o cliente.

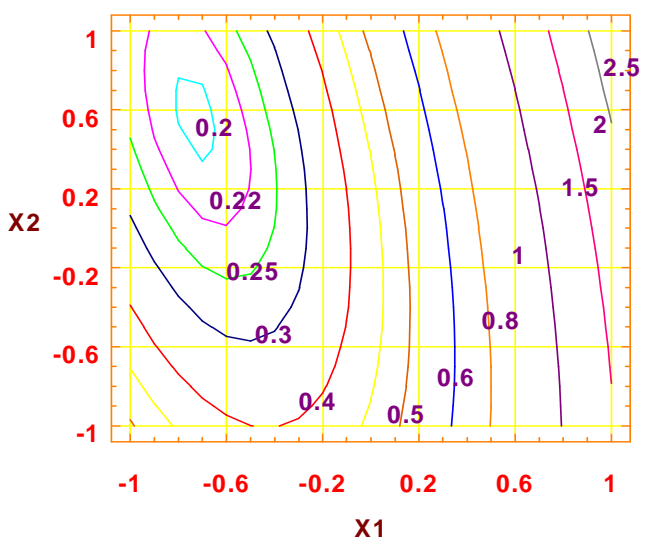

Fig. 12: Curvas de isovalores do custo da má qualidade 


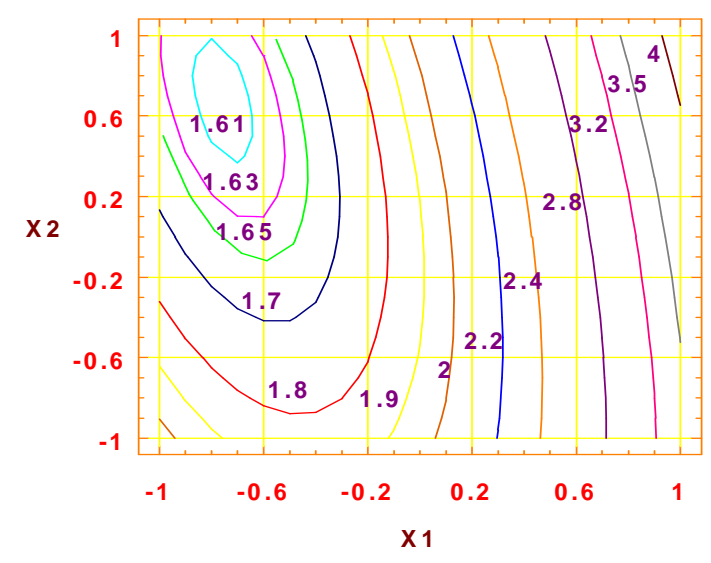

Fig. 13: Curvas de isovalores do custo global

Uma vez encontrado o ajuste ótimo, realizou-se uma análise de sensibilidade para verificar o efeito dos fatores controláveis sobre a função objetivo.

A análise de sensibilidade foi realizada da seguinte forma: variou-se um fator controlável por vez e calculou-se a função de perda, enquanto os demais fatores

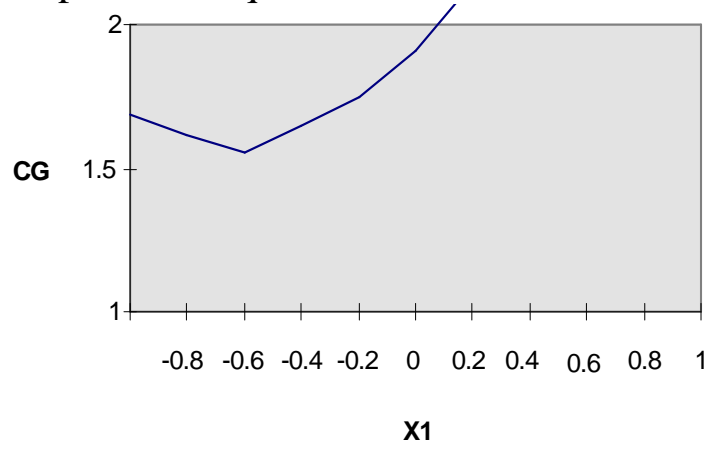

Fig. 14: Efeito de X1 sobre o custo global

Com a análise de sensibilidade foi possível verificar que apenas o fator controlável X1 exerce uma grande influência sobre a função que modela o custo global. Os demais fatores têm pouca influência sobre o custo global, comparados com o fator X1. permaneciam fixos em seus níveis ótimos. Dessa forma, foram gerados cinco gráficos distintos, nos quais a função de perda é representada em relação a cada um dos cinco fatores controláveis.

As Figuras 14 e 15 apresentam parte dos resultados da análise de sensibilidade.

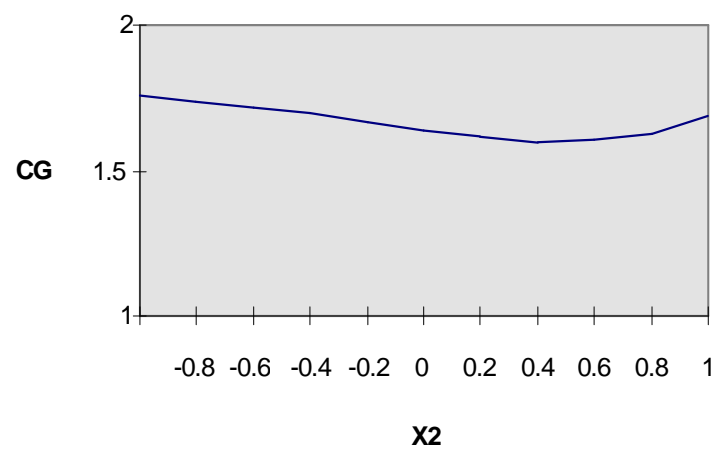

Fig. 15: Efeito de X2 sobre o custo global

$\mathrm{Na}$ Tabela 7 apresenta-se o envelope operacional que assegura uma função objetivo inferior a $\mathrm{R} \$ 1,62$ unidades monetárias

Tabela 7: Envelope Operacional para os Fatores Controláveis

\begin{tabular}{|c|c|c|}
\hline Fatores controláveis & Níveis codificados & Níveis reais \\
\hline $\mathrm{X} 1$ & $-0,80 \Leftrightarrow-0,65$ & $3,20 \Leftrightarrow 3,35$ \\
\hline
\end{tabular}




\begin{tabular}{|c|c|c|}
\hline $\mathrm{X} 2$ & $+0,40 \Leftrightarrow+1,00$ & $6,20 \Leftrightarrow 6,50$ \\
\hline $\mathrm{X} 3$ & $+0,60 \Leftrightarrow+1,00$ & $21,2 \Leftrightarrow 22,0$ \\
\hline $\mathrm{X} 4$ & $-0,40 \Leftrightarrow+1,00$ & $2,60 \Leftrightarrow 4,00$ \\
\hline $\mathrm{X} 5$ & $-1,00 \Leftrightarrow+1,00$ & $8,00 \Leftrightarrow 12,0$ \\
\hline
\end{tabular}

Uma vez feita a análise estatística completa, programou-se um simulador na linguagem QBASIC. Esse simulador fornece uma estimativa das variáveis de resposta do laboratório e do chão-de-fábrica, para qualquer ajuste dos componentes da receita, dentro dos intervalos ensaiados no experimento. A simulação é realizada a partir dos modelos individuais das variáveis de resposta, ajustados aos dados do experimento de laboratório, e dos modelos que relacionam o desempenho em laboratório e no chão-de-fábrica.

Basta entrar com um ajuste qualquer dos componentes da receita, e o simulador fornece todas as estimativas das variáveis de resposta referentes a essa receita e, ainda, o valor ideal de cada variável de resposta e suas especificações. Com isso, a empresa pode verificar previamente a eficiência de diversas receitas que posteriormente venham a ser produzidas.

O simulador fornece também as estimativas das variáveis de resposta dos ensaios realizados fora da empresa a partir dos resultados das variáveis de resposta obtidos nos ensaios realizados pela empresa.

A estimativa de algumas variáveis de resposta a partir de outras é possível, pois verificou-se que existem correlações entre as variáveis de resposta. Uma vez identificadas as correlações, modelaram-se as variáveis de resposta cujos ensaios eram feitos fora da empresa em função dos resultados dos ensaios que eram feitos pela empresa. Com esses modelos incorporados ao simulador, a empresa pode estimar todas as variáveis de resposta de uma receita a partir exclusivamente dos ensaios realizados dentro da empresa, o que reduz o custo e o tempo dos ensaios.

\section{Conclusões}

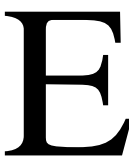

ste trabalho descreveu um estudo de otimização experimental de uma receita, realizado em uma empresa química. Nesse estudo, foram contemplados cinco fatores controláveis (componentes da mistura) e dez variáveis de resposta associadas com a qualidade do produto final.

Inicialmente quantificou-se o efeito dos fatores controláveis, ou seja, dos componentes da receita, sobre todas as variáveis de resposta separadamente. Dessa forma, a empresa pode conhecer qual o efeito de cada componente da receita sobre uma determinada variável de resposta. Ou ainda, caso a empresa necessite atingir um determinado valor para uma variável de resposta, ela saberá sobre quais fatores deverá atuar.

Verificou-se um efeito de scale-up entre as variáveis de resposta de uma receita misturada no laboratório e no chão-defábrica. Conhecendo-se esse efeito, modelaram-se as variáveis de resposta do chão-de-fábrica em função dos resultados de laboratório. Modelou-se também a variável custo em função dos componentes da receita (fatores controláveis). Uma vez obtidos os 
modelos, realizou-se a otimização considerando os valores alvo para as variáveis de resposta.

A função objetivo utilizada no estudo de otimização foi a Função de Perda Quadrática Multivariada, proposta por RIBEIRO \& ELSAYED (1995), acrescida dos custos de matéria prima e energia. Assim, a otimização foi conduzida levando em conta tanto os custos devidos a má qualidade (capturados pela função de perda de Ribeiro \& Elsayed) como os custos de małeiss prforma,emengirs.te ótimo dos fatores controláveis minimiza o custo total da receita, representando um compromisso entre o custo gerado pela má qualidade e o custo de fabricação da receita.

Posteriormente, desenvolveu-se um simulador que estima qualidade e custo para qualquer ajuste dos componentes da receita. Esse simulador pode ser usado pelos engenheiros da empresa no suporte à solução de problemas e no desenvolvimento de novas receitas.

Os resultados alcançados foram considerados extremamente positivos pela empresa, e o método de otimização foi aplicado com êxito.

\section{Referências Bibliográficas:}

BOX, G.E.P. \& DRAPER, N.: Empirical Model Building and Response Surfaces. John Wiley and Sons, Nova York, 1987.

BOX, G.E.P., HUNTER, W.G. \& HUNTER, J.S.: Statistics for experimenters, John Wiley, Nova York, 1978.

COLEMANN, D.E. \& MONTGOMERY, D.C.: “A sistematic approach to planning for a design industrial experiment", Technometrics, fevereiro, vol 35, nํ 1, pp 1-12, 1993.

ECHEVESTE, M.E. \& RIBEIRO, J.L.: "Planejando a otimização de processos", Anais do XV ENEGEP - Encontro Nacional de Engenharia de Produção, vol 1, pp 523-529, São Carlos, SP, set. 1995.

HAHN, G.J.: "Some things engineers should know about experimental design", Journal of Quality Technology, vol 9, n 1, pp 13-20, 1977.
MONTGOMERY, D.C.: Design and analysis of experiments, John Wiley and Sons, Nova York, $3^{\text {a }}$ ed., 1991.

NANNI, L. F. \& RIBEIRO, J.L.: "Planejamento e avaliação de experimentos", Caderno de Engenharia 17/87, CPGEC/UFRGS, Porto Alegre, Brasil, 2ª ed., 1991.

RIBEIRO, J.L. \& CATEN, C.S.: "Otimização experimental da qualidade: Massas cerâmicas e processo de fundição", Caderno Técnico 05/95, PPGEP/UFRGS, Porto Alegre, Brasil, 1995.

RIBEIRO, J.L. \& ELSAYED, E.A.: "A case study on process optimization using the gradient loss function", International Journal of Production Research, vol 33, n 12, pp. 3233-3248, 1995.

\section{QUALITY AND MANUFACTURING COST: A CASE STUDY IN THE CHEMICAL INDUSTRY}

\section{Abstract}

This paper presents a case study where the optimization of a chemical mixture was accomplished. The study included the following steps: problem identification, design of an experiment, model building for each individual response, choice of an objective function, and optimization. In the steps of problem identification and design of the experiment, a matrix was used to gather and to organize the information. Five control factors and ten response 
variables were included in the experiment. In the step of model building, models for mean and variability for each response variable were built. The objective function used for optimization was an extended version of the Multivariate Quadratic Loss Function proposed by Ribeiro \& Elsayed (1995). The optimization was carried out taking into account the losses due to poor quality as well as the losses due to the costs of raw materials and energy. After the optimization it was possible to identify the best set for the control factors. A sensibility analysis was performed and an operational envelop for process control is suggested.

Key words: quality, optimization, design of experiments, multiple regression, loss function. 